\title{
Results of halter cervical traction for the treatment of cervical radiculopathy: retrospective review of 81 patients
}

\author{
William C. Olivero, M.D., And Scott C. Dulebohn, M.D. \\ Department of Neurosurgery, University of Illinois College of Medicine at Peoria, Illinois; and \\ Department of Neurosurgery, University of Minnesota, Duluth, Minnesota
}

\begin{abstract}
Object. The percentage of patients responding to conservative treatment for cervical radiculopathy secondary to nerve root compression is not well quantified. To clarify this question, the authors retrospectively reviewed the records obtained over a 4-year period in patients with cervical radiculopathy to determine their response to conservative measures (cervical collar therapy and halter cervical traction).

Methods. Cervical radiculopathy was diagnosed in patients if they suffered from radiating arm pain made worse by neck movement and at least one of the following: reflex loss, dermatomal numbness, and/or myotomal weakness. Patients with neck pain alone or arm pain without neurological deficit were excluded from analysis. Those patients without excruciating pain, severe weakness, or evidence of myelopathy were offered a course of halter traction before surgery was to be considered. Ninety-six patients met the inclusion criteria; there were 61 males and 35 females, and the mean age was 47 years. Fifty-five patients presented with C-7, 37 with C-6, two with C-5, and two with C-8 radiculopathy. Eighty-one patients underwent a trial of traction that consisted of wearing a cervical collar and home-based halter cervical traction: 8 to 12 pounds, applied for 15 minutes, three times a day for 3 to 6 weeks The mean duration of symptoms prior to neurosurgical evaluation was 43 days \pm 8.3 days (standard deviation). Sixty-three (78\%) of 81 patients responded to therapeutic traction, experiencing significant or total pain relief, three could not tolerate the traction, and traction failed in 15 patients. Of the 81 patients in whom traction was undertaken, 78 underwent magnetic resonance imaging prior to being seen, which revealed herniated discs at the corresponding levels in 71 and foraminal stenosis in seven. Three of the 63 patients in whom an initial response to traction was noted suffered recurrence of symptoms and required surgery. It would appear that in patients in whom symptoms of cervical radiculopathy were present for approximately 6 weeks that $75 \%$ will respond to further conservative treatment (halter traction and cervical collar) over the next 6 weeks.
\end{abstract}

\section{KEY WORDS - cervical radiculopathy - halter traction • nerve compression}

Cervical radiculopathy is a common human ailment in which the majority of patients respond to conservative therapies. What constitutes conservative therapy and how long it should be administered are debatable. The percentage of individuals who will respond positively to conservative measures over a given period of time is also unclear. ${ }^{1,5,6,11,13}$ To clarify these questions, we typically offered home-based halter cervical traction in combination with cervical collar therapy as an option for patients with cervical radiculopathy who presented for neurosurgical evaluation. We then retrospectively reviewed their records to determine what percentage responded to these conservative measures.

Abbreviation used in this paper: $\mathrm{MR}=$ magnetic resonance.

\section{CLINICAL MATERIAL AND METHODS}

We reviewed the office records, hospital records, and imaging studies obtained in patients with cervical radiculopathy seen for neurosurgical evaluation over a consecutive 4-year period. Radiculopathy was diagnosed if the patients suffered neck and radiating arm pain aggravated by neck movement as well as at least one of the following: reflex loss, dermatomal numbness, and/or myotomal weakness. The majority of patients $(>95 \%)$ underwent neuroimaging studies, typically MR imaging, which detected pathological lesions corresponding to the nerve root involved clinically. Those patients with neck pain alone or arm pain without neurological deficit were excluded from analysis even if their imaging studies demonstrated pathological lesions. Those patients with excruciating pain, marked weakness, any significant C-5 or C-8 myotomal 
weakness, or myelopathy were not considered candidates for conservative treatment. This group represented less than $5 \%$ of patients seen during the study period. The remainder of the patients were offered a course of halter cervical traction. The device was hung over a door in the patients house, and he or she was instructed to use it three times a day for 15 minutes at a time; 8 to $12 \mathrm{lbs}$ of traction were applied. In all patients conservative measures had been previously attempted, including nonsteroidal antiinflammatory medications, narcotic analgesic agents, cervical collars, chiropractic manipulations, and modality physical therapy for various periods of time. None had attempted to use halter cervical traction. If the patients had not already been wearing a cervical collar, then one was fitted in the neutral position. Two weeks after initiation of home-based traction, patients were seen to determine if improving. If no improvement was observed the case was considered to be a therapeutic failure. If patients had experienced symptoms for at least 6 weeks, surgery was offered. If symptoms had improved completely, the patient was gradually weaned from the traction and collar therapies. If symptoms had improved somewhat, traction was continued for another 2 to 4 weeks, and the patient was then reassessed. If significant arm pain persisted, the case was considered a therapeutic failure and surgery was recommended. If traction could not be tolerated or increasing neurological deficit developed, patients were instructed to call. The mean follow-up period was 3 months after resolution of symptoms. At that time, patients were instructed to call if they suffered a recurrence of symptoms.

\section{RESULTS}

During the study period, 96 patients with cervical radiculopathy met the inclusion criteria. There were $35 \mathrm{fe}-$ males and 61 males. The mean age was 47 years. Fiftyfive patients suffered C-7, 37 C-6, two C-5, and two C-8 radiculopathy. Eighty-one patients underwent a trial of cervical collar and halter cervical traction. Of the remaining 15 patients, either they elected not to try traction or for some undocumented reason it was not offered to them. The mean duration of symptoms prior to traction therapy was $43 \pm 8.3$ days (standard deviation). There was no correlation between duration of symptoms and success of and response to therapy. Seventy-eight patients underwent cervical MR imaging prior to traction, which was typically ordered by the referring physicians. In 71 cases MR imaging revealed herniated discs at the appropriate level and seven foraminal stenoses. Normal findings or degenerative changes were demonstrated in three patients who underwent plain radiography only. There was no correlation between the size of the herniation and response to conservative therapy. Sixty-three cases were considered therapeutic successes; the patients experienced good relief of their arm pain within 2 to 6 weeks after initiation of traction and cervical collar therapy. Eighteen cases were therapeutic failures; in three of these cases, the patients could not tolerate the traction device. In three of the 63 initially successful cases, the patients suffered recurrence of their pain and underwent surgery with good results (two at 6 months and one at 9 months). The overall success rate was $74 \%$ (60 of 81 patients who underwent traction therapy). One patient with arm pain and triceps reflex loss developed index finger numbness while undergoing traction but his pain completely resolved. This probably represents the natural history of the disease. Otherwise there were no untoward traction-related effects from the cervical traction.

\section{DISCUSSION}

Neck and arm pain are common symptoms affecting as much as $50 \%$ of individuals sometime during their lives; in most cases, these symptoms will respond to conservative therapy. ${ }^{12}$ Not all of these patients will experience cervical radiculopathy because the differential diagnosis includes many other disease entities. ${ }^{4,14}$ This made evaluation of conservative treatment measures difficult prior to modern noninvasive imaging modalities, such as MR imaging. Additionally although surgery is recommended only to those patients in whom conservative therapy has failed in most surgical series, the authors rarely state what conservative therapy entailed the percentage of patients who responded to conservative measures, and the percentage ultimately requiring surgery. ${ }^{10,16,20}$

The natural history of cervical radiculopathy is difficult to define. ${ }^{4}$ The percentage of patients with nerve root compression-related cervical radiculopathy who will improve spontaneously at given periods of time after onset of symptoms is not known. In 1972, DePalma, et al., ${ }^{3}$ in an attempt to delineate the natural history, studied 68 patients in whom conservative therapy failed and who refused surgery although it was recommended; these 68 cases were compared with 281 cases in which surgery was performed. If patients involved with litigation were eliminated $63 \%$ of the surgically treated patients experienced satisfactory results and $61 \%$ of the nonsurgically treated patients experienced satisfactory results. The authors stated, however, that patients with radicular symptoms fared better when treated surgically (65\% compared with $43 \%$ ). It is unclear whether this percentage included the litigants. No statistical analysis was given. Surgically treated patients were off work a mean of 6.2 months whereas $68 \%$ of nonsurgically treated patients had returned to work within 2 months. Lees and Turner ${ }^{15}$ studied patients with cervical spondylosis, in 51 of whom there was no evidence of myelopathy, to clarify the natural history and prognosis of the disease. Of the 41 patients in the group who underwent follow up for 2 to 10 years, 19 reported no further trouble, 12 experienced slight intermittent symptoms, and 10 suffered moderate disability. Only one patient suffered a severe episode of symptoms after the first occurrence. No patient was unemployed because of spondylosis; however, the number of patients with radiculopathy is unclear, and the incidence of nerve root compression was not documented. Because the natural history of cervical radiculopathy is so ill defined, evaluation of various treatments, both conservative and surgical, is difficult.

The standard conservative treatment for cervical radiculopathy has included cervical collar therapy for immobility as well as analgesic agents for pain. Other conservative measures include chiropractic treatments, cervical trac- 


\section{Halter cervical traction}

tion, and modality physical therapy. To date, the studies performed to evaluate the various conservative measures for treatment of supposed cervical radiculopathy have had significant methodological problems: in none, for instance, has nerve root compression been documented, and neurological findings were not well described. For example, Martin and Corbin ${ }^{17}$ studied 61 patients with presumed cervical radiculopathy treated by cervical traction (30-100 lbs, 1-3 minutes twice daily) and found that $67.2 \%$ experienced definite improvement at early follow up and $77.2 \%$ experienced definite improvement at late follow up (mean 23 months). The mean duration of symptoms prior to traction, however, was not given, and radiological studies to document nerve root compression were not performed. In the cooperative study sponsored by the British Association of Physical Medicine various treatments for neck and arm pain including cervical traction were evaluated. ${ }^{2}$ They compared two groups of patients: in one group traction was applied continuously for 20 minutes, once a day, three times a week, with the patient lying supine; in the other group, patients were positioned exactly as if they were undergoing traction except that no traction was applied. Posttreatment no intergroup difference was demonstrated; in both the success rate was approximately $70 \%$. Other treatments, including modality physical therapy, cervical collars, and analgesics, were also compared with placebo tablets, untuned short-wave diathermy, and instruction in posture, and no difference among these groups was found as well. The only radiological study performed, however, was plain radiography, and therefore nerve root compression was not documented, and the majority of patients did not have neurological deficit. Goldie and Landquist ${ }^{7}$ studied 73 patients with cervical pain that radiated into the upper extremity. Nerve root compression was not documented. Paresthesias were not a common finding, and in no case was paresis present. The patients were randomly assigned to groups involving isometric exercises, cervical traction, or no physiotherapy. Although the patients who underwent traction fared slightly better, the difference was not statistically significant. Although cervical traction has been used frequently for treating cervical radiculopathy, its proponents probably equal its opponents in number. ${ }^{5,8,9,11,18,19}$ Hoff, ${ }^{11}$ for example, has stated that "generally halter traction of the cervical spine, in either the supine or sitting position, for intermittent periods during the day will relieve root pain and spasms in the neck muscles." This opinion contrasts that of Fager ${ }^{6}$ who has written that "In [his] experience, cervical traction adds nothing and, in fact, may intensify the problem."

To date, there has been no randomized prospective study in which the authors compare conservative measures to surgical intervention for cervical radiculopathy secondary to nerve root compression. One frequently cited study is that by Lunsford, et al., ${ }^{16}$ who reported on their results of anterior surgery for cervical disc disease. In their patient population, they performed anterior surgery for lateral disc herniation when conservative treatment failed to ameliorate significantly radicular symptoms within 6 weeks after initiation of therapy. Of their 253 cases, $67 \%$ experienced excellent or good results and $17 \%$ experienced fair results; overall surgery-related complications were demonstrated in $13.8 \%$, with a $23 \%$ risk of complications occurring in the patients undergoing anterior discectomy and fusion. Henderson, et al., ${ }^{10}$ reported the results of posterolateral foraminotomy for treatment of patients with cervical radiculopathy. They reviewed 846 consecutive surgically treated cases. The type of conservative therapy administered prior to surgery was not indicated. The mean duration of significant preoperative symptoms, however, was 49.5 weeks. They reported good or excellent results in $91.5 \%$ and fair or poor results in $8.5 \%$. There were no deaths, and the morbidity rate was $1.5 \%$. These figures are frequently cited when explaining the surgery-related success rate and complications to patients. Although these studies are suggestive, they obviously do not prove that surgery is better nor, if better, how much more so than conservative treatment or the natural history of the disease.

With regard to conservative treatment of cervical radiculopathy, our study is unique in several respects. All of our patients had well-documented clinical evidence of cervical radiculopathy with neurological deficit. In greater than $95 \%$ cervical MR imaging had documented nerve root compression, primarily caused by herniated cervical discs. The mean duration of symptoms prior to consultation was approximately 6 weeks, and in $95 \%$ of the patients symptoms had been present for greater than 4 weeks; additionally all patients had undergone some form of conservative treatments. We believe that because of these treatment factors, we can draw some conclusions that may benefit patients with cervical radiculopathy. Because many of our patients had undergone a trial of conservative therapy lasting 4 to 6 weeks, they would have been considered possible candidates for surgery. Obviously patients would like to know, in addition to the success rate and complication rate, their chances for improving with additional conservative treatment. Because we had no control individuals, we cannot say that the patients would not have gotten better if they had not undergone collar therapy and cervical traction. It would appear, however, that what we can realistically state the following: of patients with an approximate 6-week history of cervical radiculopathy treated by a variety of measures, who then undergo collar therapy and home-based halter cervical traction for additional 2 to 6 weeks, approximately $75 \%$ will experience resolution of their radicular pain. We believe that presenting this information is important when discussing treatment options with patients.

\section{References}

1. Boden SD, Wiesel SW: Nonoperative management of cervical disc disease, in Camins MB, O'Leary PF (eds): Disorders of the Cervical Spine. Baltimore: Williams \& Wilkins, 1992, pp 157-160 (Reference unverified)

2. British Association of Physical Medicine: Pain in the neck and arm: a multicentre trial of the effects of physiotherapy. Br Med J 1:253-258, 1966

3. DePalma AF, Rothman RH, Levitt RL, et al: The natural history of severe cervical disc degeneration. Acta Orthop Scand 43:392-396, 1972

4. Dillin W, Booth R, Cuckler J, et al: Cervical radiculopathy. A review. Spine 11:988-991, 1986 


\section{W. C. Oliverio and S. C. Dulebohn}

5. Ehni B, Ehni G, Patterson RH Jr: Extradural spinal cord and nerve root compression from benign lesions of the cervical area, in Youmans JR (ed): Neurological Surgery, ed 3. Philadelphia: WB Saunders, 1990, pp 2878-2917

6. Fager CA: Identification and management of radiculopathy. Neurosurg Clin N Am 4:1-12, 1993

7. Goldie I, Landquist A: Evaluation of the effects of different forms of physiotherapy in cervical pain. Scand J Rehabil Med 2:117-121, 1970

8. Greenfield J, Ilfeld FW: Acute cervical strain. Evaluation and short term prognostic factors. Clin Orthop 122:196-200, 1977

9. Harris PR: Cervical traction. Review of literature and treatment guidelines. Phys Ther 57:910-914, 1977

10. Henderson CM, Hennessy RG, Shuey HM Jr, et al: Posteriorlateral foraminotomy as an exclusive operative technique for cervical radiculopathy: a review of 846 consecutively operated cases. Neurosurgery 13:504-512, 1983

11. Hoff JT: Cervical disc disease and cervical spondylosis, in Wilkins RH, Rengachary SS (eds): Neurosurgery. New York: McGraw-Hill, 1985, pp 2230-2239

12. Hult L: The Munksfor Investigation. Acta Orthop Scand Suppl 16: 1959 (Reference unverified)

13. Hunt WE, Miller CA: Management of cervical radiculopathy. Clin Neurosurg 33:485-502, 1986
14. Judovich BD: Herniated cervical disc. A new form of traction therapy. Am J Surg 84:646-656, 1952

15. Lees F, Turner JWA: Natural history and prognosis of cervical spondylosis. BMJ Dec 28:1607-1610, 1963

16. Lunsford LD, Bissonette DJ, Jannetta PJ, et al: Anterior surgery for cervical disc disease. Part 1: treatment of lateral cervical disc herniation in 253 cases. J Neurosurg 53:1-11, 1980

17. Martin GM, Corbin KB: An evaluation of conservative treatment for patients with cervical disk syndrome. Staff Meet Mayo Clin 29:324-326, 1954

18. Murphey F, Simmons JCH, Brunson B: Ruptured cervical discs, 1939 to 1972. Clin Neurosurg 20:9-17, 1973

19. Rath WW: Cervical traction a clinical perspective. Orthop Rev 13:430-447, 1984 (Reference unverified)

20. Zeidman SM, Ducker TB: Posterior cervical laminoforaminotomy for radiculopathy: review of 172 cases. Neurosurgery 33: 356-362, 1993

Manuscript received December 11, 2001.

Accepted in final form January 11, 2002.

Address reprint requests to: William C. Olivero, M.D., Department of Neurosurgery, UICOMP, Box 1649, Peoria, Illinois 61656. 